\title{
Lista preliminar de hongos (Ascomycota y Basidiomycota) y mixomicetos (Myxomycota) de la Isla del Coco, Puntarenas, Costa Rica
}

\author{
Armando Ruiz-Boyer ${ }^{1 *} \&$ Alexánder Rodríguez-González ${ }^{1}$ \\ 1. Departamento de Historia Natural, Museo Nacional de Costa Rica, San José, Costa Rica; aruiz@museocostarica.go.cr, \\ arodriguez@museocostarica.go.cr \\ * Correspondence
}

Recibido 30-I-2019. Corregido 26-IV-2019. Aceptado 25-VI-2019.

\begin{abstract}
Preliminary list of fungi (Ascomycota and Basidiomycota) and myxomycetes (Myxomycota) of Isla del Coco, Puntarenas, Costa Rica. Introduction: The inventory of fungi and myxomycetes on Cocos Island is a relatively recent process. The first record of fungi was published by Cooke \& Bonar (1961), followed by the listings of Fosberg \& Klawe (1966), Gómez (1983), and the contributions of Mata (2003) and Mata, Halling, \& Mueller (2003). Subsequent to these studies on fungi, Rojas \& Stevenson (2008) carried out research on the diversity, ecology and distribution of myxomycetes. Objective: To present a compilation and taxonomic update of the inventory of fungi and myxomycetes that were registered on Cocos Island and to facilitate the knowledge about their diversity on the island. Methods: The list provided here is based on the review of available literature, databases of herbaria and on the determination of the specimens collected recently by several researchers of the Department of Natural History of the National Museum of Costa Rica. Three field trips were made to the island to collect and photograph specimens. These field trips took place between 2016 and 2017, in the months of June, November and December. The names of the species included were adjusted according to the online database Index Fungorum. Results: A total of 648 records distributed in 401 fungi (mainly macrofungi) and 247 myxomycetes were analyzed for Cocos Island. This number of records represent 185 species, of which 139 correspond to fungi and 46 to myxomycetes. The fungi registered were distributed in 85 genera, 46 families (four of them under the term "Incertae sedis"), 21 orders (two of them as "Incertae sedis") and two divisions (Ascomycota and Basidiomycota); meanwhile, the myxomycetes were classified in 21 genera, 10 families, 5 orders and one division (Myxomycota). The family represented most in Ascomycota was Xylariaceae (8 spp.), followed by Pyronemataceae ( 3 spp.) and Sarcoscyphaceae ( 2 spp.). In Basidiomycota, the most diverse families were Omphalotaceae and Polyporaceae (both had $11 \mathrm{spp}$.), followed by Marasmiaceae (10 spp.), Agaricaceae (8 spp.), Hymenochaetaceae (8 spp.), Mycenaceae (8 spp.), Ganodermataceae (6 spp.) and Meripilaceae (6 spp.). The families with the highest number of species recorded in Myxomycota were Stemonitidaceae (14 spp.), Physaraceae (8 spp.), Trichiaceae (6 spp.), Didymiaceae (5 spp.) and Arcyriaceae (4 spp.). Thirty-four species were recorded for the first time for Cocos Island, the majority of them belonging to fungi (Ascomycota, $8 \mathrm{spp}$. and Basidiomycota, $21 \mathrm{spp}$.) and the remaining species (5) to myxomycetes. On the island, some of the species registered are relevant because of their edible or medicinal properties. Photographs of some of the fungi collected on the island are included. Conclusions: The list generated in this study is still preliminary for the fungi and myxomycetes that were registered on Cocos Island due to certain groups such as microfungi being scarcely collected.
\end{abstract}

Key words: Ascomycota; Basidiomycota; Myxomycota; neotropics; Cocos Island; inventory.

Ruiz-Boyer, A. \& Rodríguez-González, A. (2020). Lista preliminar de hongos (Ascomycota y Basidiomycota) y mixomicetos (Myxomycota) de la Isla del Coco, Puntarenas, Costa Rica. Revista de Biología Tropical, 68(Supl. 1), S33-S56. 
En Costa Rica, la Isla del Coco representa la segunda isla más grande $\left(24 \mathrm{~km}^{2}\right)$, luego de Chira $\left(43 \mathrm{~km}^{2}\right)$ en el Golfo de Nicoya (Herrera, 1985) y se encuentra a una distancia de tierra continental de $548 \mathrm{~km}$ de la costa pacífica del país (Hammel, Grayum, Herrera, \& Zamora, 2004). Forma parte del Área de Conservación Marina Cocos (ACMC) del Sistema Nacional de Áreas de Conservación (SINAC), del Ministerio de Ambiente y Energía (MINAE). Fue declarada Parque Nacional debido a su belleza escénica y valor biológico (Gómez, 1983), según el Decreto Ejecutivo No. 8748-A, del 22 de junio de 1978.

El inventario sobre la diversidad de hongos y mixomicetos en la Isla del Coco es un proceso relativamente reciente en comparación con el de plantas, este último inició con los primeros registros en 1684 con el bucanero británico William Dampier (Hammel et al., 2004), aunque una primera lista sistemática fue publicada hasta muchos años después por Rose (1892). De acuerdo con la literatura disponible, un primer registro de hongos en la Isla del Coco fue publicado por Cooke y Bonar (1961), en el artículo titulado "Additional fungi from the Galápagos and other pacific costal islands collected during the Templeton Crocker Expedition, 1932". En este trabajo dichos autores incluyeron la especie Poria umbrinella Bres., actualmente conocida como Fulvifomes umbrinellus (Bres.) Y.C. Dai, que fue recolectada sobre madera podrida en el año de 1905 por el botánico estadounidense Alban Stewart e identificada años más tarde por el micólogo Josiah Lincoln Lowe.

La primera lista de hongos para la isla fue dada a conocer por Fosberg y Klawe (1966), quienes se basaron en especímenes recolectados por el mismo Klawe en el año 1964 y en la revisión de la literatura existente a la fecha. En esa lista se presentó un inventario preliminar que, además de hongos, incluía espermatófitas, helechos, musgos, líquenes y hepáticas, aunque para el caso particular de hongos, esta publicación solo citó 10 especies, sin indicar especímenes de referencia ni tampoco herbario(s) donde fueron depositados los ejemplares.

Una segunda lista fue presentada por Gómez (1983) como resultado de sus 11 expediciones a la isla. El autor enumeró 85 especies (Basidiomycetes y Ascomycetes) distribuidas en 24 familias e indicó que su inventario podría representar alrededor del 50\% de las especies de hongos presentes en la isla, y aunque no citó especímenes de referencia menciona que la mayoría fueron depositados en el Herbario del Real Jardín Botánico Kew, Londres, Inglaterra $(\mathrm{K})$ y algunos otros en el Museo Field de Historia Natural, Chicago, Estados Unidos (F) y en el Herbario Nacional de Costa Rica (CR).

Otro aporte a los hongos de la isla fue realizado por Mata (2003) y Mata, Halling y Mueller (2003). Estos investigadores en sus guías de campo sobre los Macrohongos de Costa Rica incluyeron siete especies de Basidiomycota, una de las cuales [Stereum ostrea (Blume \& T. Nees) Fr.] no había sido citada en las publicaciones previamente mencionadas.

Posterior a los trabajos anteriores sobre hongos fue llevada a cabo una investigación sobre la diversidad, ecología y distribución de mixomicetos, tradicionalmente estudiados por los micólogos (Piepenbring \& Ruiz-Boyer, 2008), a lo largo de una gradiente altitudinal en la isla (Rojas \& Stephenson, 2008). En total fueron recolectados 241 especímenes que se distribuyeron en 41 especies. Los ejemplares fueron obtenidos de sustratos de humus terrestre o aéreo, así como de corteza de troncos y ramas de árboles.

Como resultado de tres expediciones, de la revisión de la literatura disponible, de la consulta de bases de datos de herbarios y de la identificación de especímenes recolectados recientemente por varios investigadores del Departamento de Historia Natural del Museo Nacional de Costa Rica, se presenta una recopilación y actualización taxonómica del inventario de hongos y mixomicetos que han sido recolectados en la Isla del Coco, de tal manera que facilite el conocimiento sobre la diversidad de dichos grupos en la isla. 


\section{MATERIALES Y MÉTODOS}

Sitio de estudio: El presente trabajo se realizó en la Isla del Coco, provincia de Puntarenas, ubicada entre las coordenadas geográficas $5^{\circ} 30^{\prime} 06^{\prime \prime}-5^{\circ} 33^{\prime} 26^{\prime \prime} \mathrm{N}$ y $87^{\circ} 01^{\prime} 47^{\prime}$ ' - 8705’46” W (Montoya, 2007). La topografía irregular de la isla, con elevaciones que van de 0 a $575.5 \mathrm{~m}$, y la alta precipitación, con un promedio anual de 5000 a $7000 \mathrm{~mm}$ que es mayor en el punto más alto, Cerro Iglesias (Montoya, 2005), contribuyen a una cobertura relativamente diversa de bosque pluvial premontano (Tosi, 1969). El clima se caracteriza por la nubosidad abundante y alta precipitación durante todo el año, los meses más secos van de enero a marzo (Herrera, 1985). La temperatura promedio anual varía entre $24{ }^{\circ} \mathrm{C}$ y $26{ }^{\circ} \mathrm{C}$ (Montoya, 1990).

Lista preliminar: La presente lista incluye todas las especies de hongos y mixomicetos que han sido registradas hasta la fecha para la Isla del Coco. La información de este inventario se obtuvo principalmente mediante la búsqueda de los especímenes recolectados en la Isla del Coco, la mayoría depositados en el Herbario Nacional de Costa Rica (CR) y en menor medida en el Herbario del Museo Field de Historia Natural (F) y el Herbario de la Academia de Ciencias de California (CAS). Además, se realizó una revisión de las publicaciones previas que enlistan las especies de hongos y mixomicetos de la isla, esto incluyó los trabajos de Cooke y Bonar (1961), Fosberg y Klawe (1966), Gómez (1983), Mata (2003), Mata et al. (2003) y Rojas y Stephenson (2008).

Adicionalmente, el segundo de los autores y otros investigadores del Departamento de Historia Natural del Museo Nacional de Costa Rica realizaron tres giras de campo a la isla para recolectar y fotografiar especímenes. Estas giras se llevaron a cabo entre los años 2016 y 2017, en los meses de junio, noviembre y diciembre. Los ejemplares producto de las giras de campo fueron depositados en el Herbario CR. La Figura 1 muestra los puntos donde se han recolectado especímenes de hongos y mixomicetos en la isla, según la información registrada en la base de datos del Herbario CR.

Los ejemplares se recolectaron al azar a partir de recorridos por el sotobosque, bordes de caminos y senderos. Se examinaron troncos en pie, caídos o en descomposición y el suelo. Se recolectaron todas las especies de macrohongos y mixomicetos observadas durante los recorridos. El material recolectado se colocó, según sus peculiaridades, en bolsas de papel o en papel encerado y se tomó nota de sus características macroscópicas en estado fresco, así como del sustrato en el que se encontraron. Posteriormente, fueron llevados al Herbario CR para completar su preservación e identificación. La preservación se realizó por secado en estufa a $30^{\circ} \mathrm{C}$ aproximadamente durante 1 ó 2 días, de acuerdo con la consistencia de cada ejemplar. Para la identificación de las muestras de hongos recolectadas recientemente (Ascomycota y Basidiomycota) se siguió la metodología empleada por CarranzaVelázquez, Marín-Méndez, Ruiz-Boyer y Di Stéfano-Gandolfi (2014); mientras que para mixomicetos (Myxomycota) se hizo de acuerdo con Stephenson y Stempen (1994). También, se realizó por comparación con muestras del Herbario CR cuando las características que presentaron los ejemplares no coincidieron con ninguna de las descripciones empleadas.

Las fotografías de los ejemplares recolectados, se utilizaron como material de apoyo en la identificación. Las imágenes capturadas se catalogaron y fueron incorporadas a la colección de fotografías del Departamento de Historia Natural del Museo Nacional de Costa Rica.

La nomenclatura y el estatus taxonómico de los nombres científicos incluidos en este inventario fue revisado y actualizado según lo propuesto en la base de datos en línea Index Fungorum (Recuperado de http://www. indexfungorum.org/Names/Names.asp; 2018). La sinonimia (Sin.), cuando se presentó, es indicada entre paréntesis cuadrados posterior al nombre actual de la especie.

Las especies que no fueron ubicadas en un orden o en una familia debido a que su clasificación actual es incierta, según el Index 


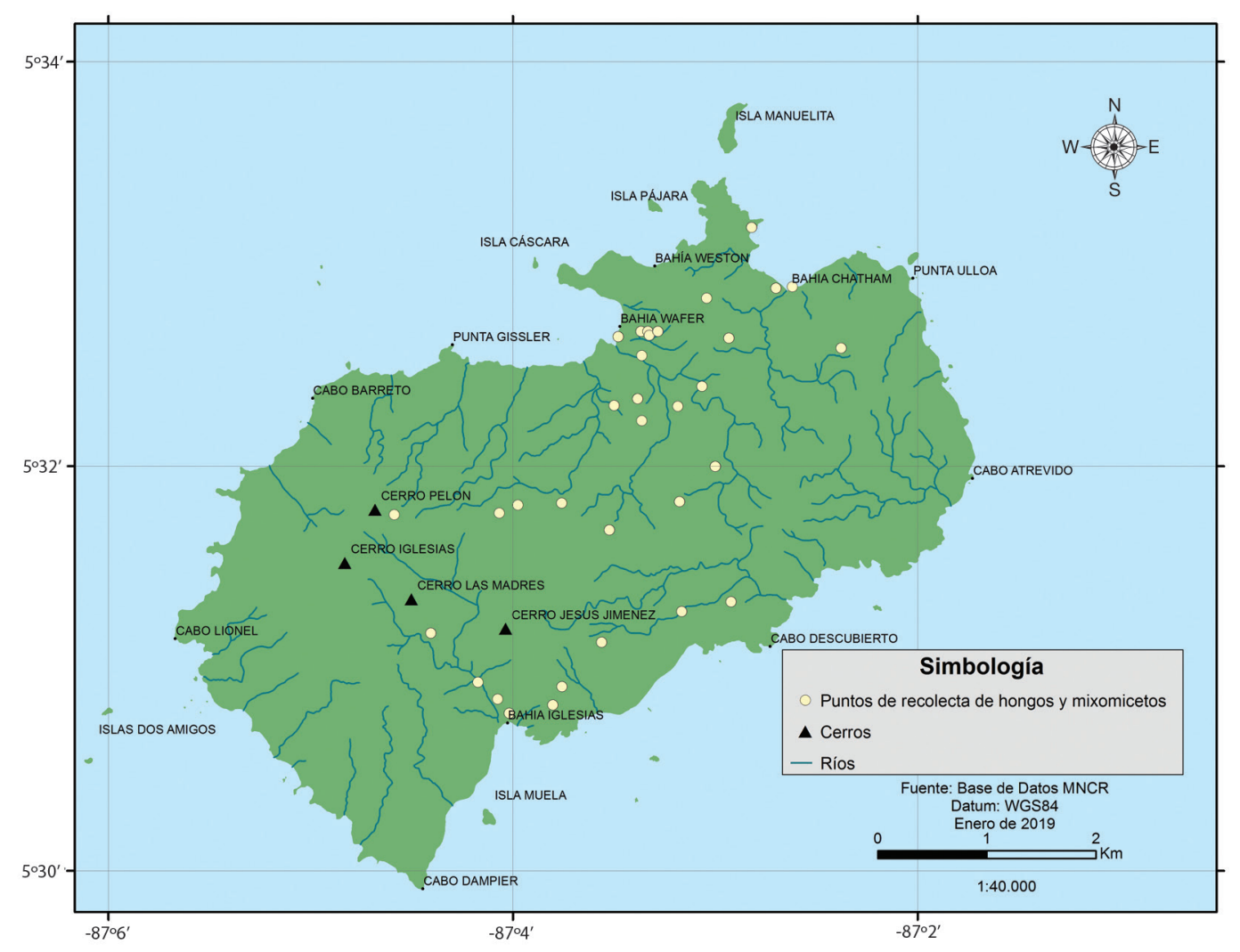

Fig. 1. Puntos de recolección de hongos y mixomicetos en la Isla del Coco (Puntarenas, Costa Rica).

Fig. 1. Fungi and myxomycetes collecting points on Cocos Island (Puntarenas, Costa Rica).

Fungorum, fueron especificadas en la lista con la expresión latina "Incertae sedis". La lista de especies incluida en este inventario se presenta ordenada por categoría de división, es decir, por Ascomycota, Basidiomycota y Myxomycota. A la vez, en cada división se enlistan los órdenes, las familias, los géneros y las especies correspondientes, todos ordenados alfabéticamente según sea el caso. A cada especie se le asignó: autor (es), ejemplar testigo (inicial del nombre y primer apellido del recolector, número consecutivo de recolección y acrónimo del herbario donde se encuentra depositado). En caso de que no se localizara un testigo para la especie, se indica el autor (es) que la registró, seguido por la abreviación s.n. (sin número). Los acrónimos de los herbarios se anotan de acuerdo con Holmgren, Holmgren y Barnett (1990).
Las especies incluidas en los trabajos de Cooke y Bonar (1961), Fosberg y Klawe (1966), Gómez (1983), Mata (2003), Mata et al. (2003) y Rojas y Stephenson (2008) se identifican en el Apéndice con los números en superíndice 1, 2, 3, 4, 5 у 6, respectivamente. Los taxones no señalados con dichos números e identificados a nivel de especie representan nuevos registros para la isla. Además, la lista proporciona información sobre el número de ejemplares que fueron recopilados para cada especie.

\section{RESULTADOS}

Un total de 648 registros distribuidos en 401 hongos (principalmente macrohongos) y 247 mixomicetos fueron analizados para la Isla 
del Coco. El 15.7\% de los registros de hongos y el $0.4 \%$ de los registros de mixomicetos fueron recolectados durante el desarrollo de esta investigación. La totalidad de registros recopilados representan 185 especies, de las cuales 139 corresponden a hongos $(75.1 \%)$ y 46 a mixomicetos (24.9\%) (ver Apéndice).

Los hongos listados se distribuyeron en 85 géneros, 46 familias (cuatro de ellas bajo el término "Incertae sedis"), 21 órdenes (dos de ellos como "Incertae sedis") y dos divisiones (Ascomycota y Basidiomycota). Los mixomicetos encontrados se clasificaron en 21 géneros, 10 familias, 5 órdenes y una división (Myxomycota). De acuerdo con el total de taxones recopilados, la división Basidiomycota (hongos) fue la más diversa en la isla con 118 especies $(63.8 \%$ del total registrado), seguida por Myxomycota (mixomicetos, 46 spp., 24.9\%) y Ascomycota (hongos, 21 spp., $11.3 \%$ ).

La mayor parte de las especies en Ascomycota se ubicaron en el orden Xylariales (9 spp.), seguido por Pezizales (6 spp.) y Pleosporales (2 spp.). En el caso de Basidiomycota las especies se distribuyeron especialmente en el orden Agaricales (55 spp.), seguido por los Polyporales (29 spp.), Hymenochaetales (8 spp.), Cantharellales (5 spp.), Auriculariales y Russulales (4 spp. cada uno), Geastrales (3 spp.) y Dacrymycetales, Thelephorales y Tremellales ( 2 spp. cada uno). En tanto que en Myxomycota las especies se situaron principalmente en el orden Stemonitales (14 spp.), seguido por Physarales (13 spp.), Trichiales (10 spp.), Liceales (6 spp.) y Echinosteliales (3 spp.).

En la división Ascomycota la familia Xylariaceae con 8 especies fue la más diversa, seguida por Pyronemataceae (3 spp.) y Sarcoscyphaceae (2 spp.). Además, se registraron dos taxones [Rimaconus jamaicensis (Seaver) Huhndorf, F.A. Fernández, Joanne E. Taylor \& K.D. Hyde y Xenolophium pachythele (Berk. \& Broome) Huhndorf], que fueron ubicados bajo el término "Incertae sedis" de acuerdo con el Index Fungorum. En Basidiomycota las familias mejor representadas fueron Omphalotaceae y Polyporaceae, ambas tienen 11 especies, seguidas por Marasmiaceae (10 spp.), Agaricaceae, Hymenochaetaceae y Mycenaceae (8 spp. cada una) y Ganodermataceae y Meripilaceae (6 spp. cada una). En esta última división se registraron tres taxones, Camarophyllopsis dennisiana (Singer) Arnolds, Panaeolus aff. antillarum (Fr.) Dennis y P. semiovatus (Sowerby) S. Lundell \& Nannf., que fueron situados dentro de la expresión latina "Incertae sedis". Por su parte, en Myxomycota el mayor número de especies se ubicaron en las familias Stemonitidaceae (14 spp.), Physaraceae (8 spp.), Trichiaceae (6 spp.), Didymiaceae (5 spp.) y Arcyriaceae (4 spp.).

Los géneros más diversos en la isla en el caso de los hongos, en orden descendente (4 ó más especies), fueron Marasmiellus (Omphalotaceae, Basidiomycota) con 8 spp., Rigidoporus (Meripilaceae, Basidiomycota) y Xylaria (Xylariaceae, Ascomycota) con 6 spp. respectivamente, Ganoderma (Ganodermataceae, Basidiomycota), Marasmius (Marasmiaceae, Basidiomycota) y Mycena (Mycenaceae, Basidiomycota) con 5 spp. cada uno. En tanto que en mixomicetos fueron Physarum (Physaraceae) con 6 spp., y Arcyria (Arcyriaceae) y Comatricha (Stemonitidaceae) con $4 \mathrm{spp}$.

Del total de registros recopilados en este inventario, 34 taxones se listan solo a nivel de género. Entre estos registros, 17 fueron recolectados recientemente por funcionarios del Departamento de Historia Natural del Museo Nacional de Costa Rica y corresponden a hongos [Camillea, Collybia, Cotylidia, Flammulina, Gymnopus, Kretzschmaria, Marasmius, Pleurotus, Rigidoporus (tres taxones), Scutellinia, Xylaria (cuatro taxones) y Xylobotryum]; cuatro se asocian con otros recolectores ( $\mathrm{Cla}$ vulina, Ganoderma, Hydnum y Phellinus) y uno (Licea) que pertenece a un mixomiceto y fue comunicado por Rojas y Stephenson (2008). Todos estos taxones están disponibles en las colecciones del Herbario CR. Por otro lado, los restantes 12 son aportes de Luis Diego Gómez [Gerronema (dos taxones) Hydropus, Lactocollybia, Lepiota (dos taxones), Marasmiellus (tres taxones), Panellus, Stereum y Thelephora]. Según el mismo Gómez (1983) 
sus colecciones de hongos de la Isla del Coco fueron depositadas en el Herbario del Real Jardín Botánico Kew (K) y algunos otros en el Museo Field de Historia Natural (F) y en el Herbario CR. Sin embargo, en este estudio se comprobó que no existen especímenes de la Isla del Coco recolectados por Luis Diego Gómez en el acervo del Herbario CR.

Del total de especies incluidas en el presente catálogo, 34 se comunican por primera vez para la Isla del Coco. La mayor parte de estas especies corresponden a hongos de la división Basidiomycota (21 spp., 61.8\% del total), seguida por Ascomycota (hongos, 8 spp., $23.5 \%$ ) y Myxomycota (mixomicetos, 5 spp., 14.7\%). La tabla 1 presenta la lista de especies que resultaron registros nuevos para la isla. Las especies marcadas con un asterisco (*) representan taxones que fueron recolectados durante las expediciones recientemente realizadas por curadores del Departamento de Historia Natural del Museo Nacional de Costa Rica.

Entre los hongos de la división Ascomycota mejor recolectados en la isla (con no menos de cuatro ejemplares) están: Xylaria arbuscula y $X$. polymorpha (4 ejemplares). En tanto que en Basidiomycota se encuentran: Rigidoporus microporus (7 ejemplares), Ganoderma cf. lucidum, Trametes elegans y T. hydnoides (6 ejemplares), Favolus tenuiculus y Ganoderma cf. applanatum (5 ejemplares), Auricularia auricula-judae, Earliella scabrosa, Hymenochaete iodina, Lentinus crinitus y L. tricholoma (4 ejemplares). En el caso de Myxomycota figuran: Arcyria cinerea (44 ejemplares), Cribraria microcarpa (17 ejemplares), Collaria arcyrionema (14 ejemplares), Perichaena chrysosperma (10 ejemplares), Comatricha tenerrima (9 ejemplares), Cribraria violacea (8 ejemplares), Collaria lurida (7 ejemplares), C. elegans (6 ejemplares), Lamproderma scintillans (5 ejemplares) y Physarum melleum (4 ejemplares). Véase el Apéndice, para conocer la cantidad total de ejemplares recopilados para cada especie incluida en este trabajo.

El presente inventario de hongos y mixomicetos de la Isla del Coco es el resultado del aporte de diversos investigadores y/o recolectores. De acuerdo con la información recopilada sobresalen por la cantidad de ejemplares documentados en orden descendente: Carlos Rojas (245 ejemplares, Myxomycota), Eida Fletes (204 ejemplares, Ascomycota y Basidiomycota), Luis Diego Gómez (89 ejemplares, Ascomycota y Basidiomycota), Alexander Rodríguez (48 ejemplares, Ascomycota y Basidiomycota), Frederick Ball (32 ejemplares, Basidiomycota) y Armando Estrada (12 ejemplares, Ascomycota y Basidiomycota).

\section{DISCUSIÓN}

Ávalos (2019), menciona que el número de hongos en Costa Rica es de 3000 especies; sin embargo, indica que esta cifra es meramente especulativa ya que se basa en la opinión de expertos, debido a que el inventario de este grupo de organismos todavía está incompleto en el país. Con base en esta información y en los datos recopilados para este trabajo, los hongos registrados en la Isla del Coco representan un $4.6 \%$ del total estimado para Costa Rica. En el caso de los mixomicetos, Rojas, Rojas y Lado (2018) indican que actualmente se conocen para el país un total de 242 especies, de las cuales hasta la fecha 46 de ellas (19\%) se encuentran en la isla.

De acuerdo con Rojas y Stephenson (2008), el viento y la composición de la comunidad de plantas en la isla parecen ser los factores más importantes que determinan la aparición de los mixomicetos. Posiblemente, ambos componentes también puedan favorecer el desarrollo de los hongos, puesto que muchos de ellos utilizan la madera de las plantas, viva o en descomposición, como sustrato. Se estima que la flora de la Isla del Coco se compone de 294 especies de plantas vasculares (Estrada, Sánchez, \& Rodríguez, en prep.), que podrían beneficiar el crecimiento de los hongos. Otro factor determinante en la formación de los cuerpos fructíferos de los hongos es el clima, del cual dependen estrechamente, ya que necesitan de una cantidad adecuada de agua y una temperatura promedio entre $22^{\circ} \mathrm{C}$ y $28^{\circ} \mathrm{C}$ para poder desarrollarse (Carranza-Velázquez, Marín-Méndez, 
TABLA 1

Nuevos registros de hongos (Ascomycota y Basidiomycota) y mixomicetos (Myxomycota) para la Isla del Coco (Puntarenas, Costa Rica)

TABLE 1

New fungi (Ascomycota and Basidiomycota) and myxomycetes (Myxomycota) records for Cocos Island (Puntarenas, Costa Rica)

\begin{tabular}{|c|c|c|}
\hline DIVISIÓN / Familia & Especie & Testigo \\
\hline \multicolumn{3}{|l|}{ ASCOMYCOTA } \\
\hline Melanommataceae & Byssosphaeria jamaicana (Sivan.) M.E. Barr & E. Fletes 7730 (CR) \\
\hline Chaetosphaeriaceae & $\begin{array}{l}\text { Chaetosphaeria raciborskii (Penz. \& Sacc.) F.A. Fernández } \\
\text { \& Huhndorf }\end{array}$ & E. Fletes 7765 (CR) \\
\hline Leptosporellaceae & Leptosporella gregaria Penz. \& Sacc. & E. Fletes 7762 (CR) \\
\hline Incertae sedis & $\begin{array}{l}\text { Rimaconus jamaicensis (Seaver) Huhndorf, F.A. Fernández, } \\
\text { Joanne E. Taylor \& K.D. Hyde }\end{array}$ & E. Fletes 7618 (CR) \\
\hline Xylariaceae & Rosellinia arcuata Petch & E. Fletes 7685 (CR) \\
\hline Incertae sedis & Xenolophium pachythele (Berk. \& Broome) Huhndorf & E. Fletes 7751 (CR) \\
\hline Xylariaceae & Xylaria arbuscula Sacc. & E. Fletes 7728 (CR) \\
\hline Xylariaceae & Xylaria polymorpha (Pers.) Grev. * & A. Rodríguez 14630 (CR) \\
\hline \multicolumn{3}{|l|}{ BASIDIOMYCOTA } \\
\hline Auriculariaceae & Auricularia auricula-judae (Bull.) Quél. * & A. Rodríguez 14776 (CR) \\
\hline Clavariaceae & Clavulinopsis fusiformis (Sowerby) Corner * & A. Rodríguez 14808 (CR) \\
\hline Clavariaceae & Clavulinopsis laeticolor (Berk. \& M.A. Curtis) R.H. Petersen & L. Gómez 18032 (F) \\
\hline Meruliaceae & Flaviporus liebmannii (Fr.) Ginns & E. Fletes 7636 (CR) \\
\hline Ganodermataceae & Ganoderma cf. australe (Fr.) Pat. & E. Fletes 7607 (CR) \\
\hline Ganodermataceae & Ganoderma cf. lucidum (Curtis) P. Karst. * & A. Rodríguez 14783 (CR) \\
\hline Ganodermataceae & Ganoderma stipitatum (Murrill) Murrill & E. Fletes 7619 (CR) \\
\hline Geastraceae & Geastrum schweinitzii (Berk. \& M.A. Curtis) Zeller & E. Fletes 7651 (CR) \\
\hline Hymenochaetaceae & Hymenochaete iodina (Mont.) Baltazar \& Gilbertoni & E. Fletes 7634 (CR) \\
\hline Agaricaceae & Lycogalopsis solmsii E. Fisch. & E. Fletes 7624 (CR) \\
\hline Agaricaceae & Lycoperdon fuligineum Berk. \& M.A. Curtis & E. Fletes $7642(\mathrm{CR})$ \\
\hline Omphalotaceae & Marasmiellus cocosensis Singer & L. Gómez 17974 (F) \\
\hline Omphalotaceae & Marasmiellus pernambucensis Singer & L. Gómez 17969 (F) \\
\hline Hymenochaetaceae & Phellinus gilvus (Schwein.) Pat. & E. Fletes 7804 (CR) \\
\hline Inocybaceae & Pleuroflammula squarrulosa Singer & L. Gómez 17998 (F) \\
\hline Polyporaceae & Polyporus dictyopus Mont. & E. Fletes 7801 (CR) \\
\hline Meripilaceae & Rigidoporus microporus (Sw.) Overeem * & A. Estrada 6155 (CR) \\
\hline Meripilaceae & Rigidoporus ulmarius (Sowerby) Imazeki & E. Fletes 7879 (CR) \\
\hline Polyporaceae & Tinctoporellus epimiltinus (Berk. \& Broome) Ryvarden & E. Fletes 7603 (CR) \\
\hline Polyporaceae & Trametes hydnoides (Sw.) Fr. * & A. Rodríguez 14821 (CR) \\
\hline Tremellaceae & Tremella fuciformis Berk. * & A. Rodríguez 14812 (CR) \\
\hline \multicolumn{3}{|l|}{ MYXOMYCOTA } \\
\hline Arcyriaceae & Arcyria denudata (L.) Wettst. & S. Stephenson 18363 (CR) \\
\hline Trichiaceae & Hemitrichia pardina (Minakata) Ing & C. Rojas 990 (CR) \\
\hline Stemonitidaceae & Lamproderma arcyrioides (Sommerf.) Rostaf. & C. Rojas 982 (CR) \\
\hline Tubiferaceae & Lycogala epidendrum (J.C. Buxb. ex L.) Fr. & C. Rojas 1978 (CR) \\
\hline Physaraceae & Physarella oblonga (Berk. \& M.A. Curtis) Morgan & C. Rojas 1476 (CR) \\
\hline
\end{tabular}


Ruiz-Boyer, \& Di Stefano-Gandolfi, 2017). Variaciones semejantes de temperatura han sido comunicadas para la Isla del Coco por Montoya (1990), que menciona fluctuaciones entre $24^{\circ} \mathrm{C}$ y $26^{\circ} \mathrm{C}$ de promedio anual.

Es posible que algunas otras especies de hongos y mixomicetos puedan estar presentes en la Isla del Coco, ya que constituyen taxones muy comunes en el territorio continental de Costa Rica y en el Neotrópico. Por ejemplo, entre los hongos estarían especies como Auricularia mesenterica, Cookeina tricholoma, Filoboletus gracilis, Hexagonia glabra, Phillipsia domingensis, Pycnoporus sanguineus y Schizophyllum commune. Entre los mixomicetos podrían encontrarse Arcyria insignis, Ceratiomyxa fruticulosa var. fruticulosa, Didymium bahiense, D. nigripes, Fuligo septica, Hemitrichia calyculata, Metatrichia floriformis, Physarum cinereum y P. didermoides; taxones que de acuerdo con Rojas et al. (2018) han sido documentadas en las siete provincias del país.

Según la información recopilada de la base de datos del Herbario CR, todas las especies de hongos y mixomicetos registradas en el presente estudio, incluyendo los nuevos registros, han sido recolectadas en diferentes localidades de Costa Rica continental. A pesar de ello, se considera que las especies listadas representan un insumo importante de información para futuros estudios sobre taxonomía, distribución y riqueza de especies en territorio insular del país y en particular de la Isla del Coco.

Hasta el momento, son escasas las especies de la división Ascomycota que se han registrado en la Isla del Coco $(15.1 \%$ del total de hongos), en comparación con Basidiomycota $(84.9 \%)$. Es posible que estos resultados se deban a que la división Ascomycota incluye órdenes con especies que poseen cuerpos fructíferos de tamaños muy pequeños, difíciles de observar a simple vista, por lo que muchas veces pasan desapercibidos para los no especialistas (Carranza-Velázquez et al. 2014). Asimismo, es muy probable que este bajo porcentaje esté relacionado a que dicha división incluye la mayor parte de los llamados microhongos, los cuales han sido poco estudiados en Costa
Rica (Chaverri, Huhndorf, Rogers, \& Samuels, 2010) y cuya representación en este inventario es de tan solo un $7.9 \%$. Situación similar ocurre con los mixomicetos registrados (24.9\%), donde la mayoría de las especies tienden a ser insconspicuas o esporádicas y no siempre son fáciles de detectar en el campo (Stephenson, Schnittler, \& Novozhilov, 2008).

Los datos recopilados en este inventario para hongos son muy semejantes a los mencionados por Carranza-Velázquez et al. (2014) en un bosque tropical secundario de zona baja. La investigación determinó la riqueza de macrohongos y fue realizada en La Estación La Leona del Parque Nacional Corcovado, Puntarenas, Costa Rica. Los autores encontraron que los órdenes mejor representados corresponden principalmente a los Polyporales (50 spp.) y los Agaricales (36 spp.) en Basidiomycota y a los Xylariales (29 spp.) y los Pezizales (6 spp.) en Ascomycota. En la Isla del Coco el mayor número de especies registradas también se agruparon en los órdenes antes mencionados (ver Apéndice). Estos órdenes constituyen los más conspicuos entre los hongos y uno de ellos, los Polyporales, son los mejor conocidos en Costa Rica (Carranza \& Ruiz-Boyer, 2005; Mata, Ruiz-Boyer, Carranza, \& Ryvarden, 2007; Decock \& Ryvarden, 2013).

Varios de los ejemplares producto de las recolectas realizadas por los curadores del Departamento de Historia Natural del Museo Nacional de Costa Rica se listan únicamente identificados a nivel de género (e.g., Camillea, Gymnopus, Marasmius, Phellinus, Rigidoporus, Scutellinia, Xylaria y Xylobotryum). No obstante, por las características que poseen se considera que estos taxones pueden representar especies diferentes a las ya registradas para la isla. Actualmente, estos ejemplares se encuentran en estudio, por lo que el número de especies de hongos en la isla podría eventualmente aumentar.

Con base en la literatura revisada (Guzmán, 1997; Franco-Molano, Vasco-Palacios, López-Quintero, \& Boekhout, 2005; Guzmán \& Piepenbring, 2011; Carranza-Velázquez et al. 2017; Rodríguez-Palma, Montoya, Kong, 

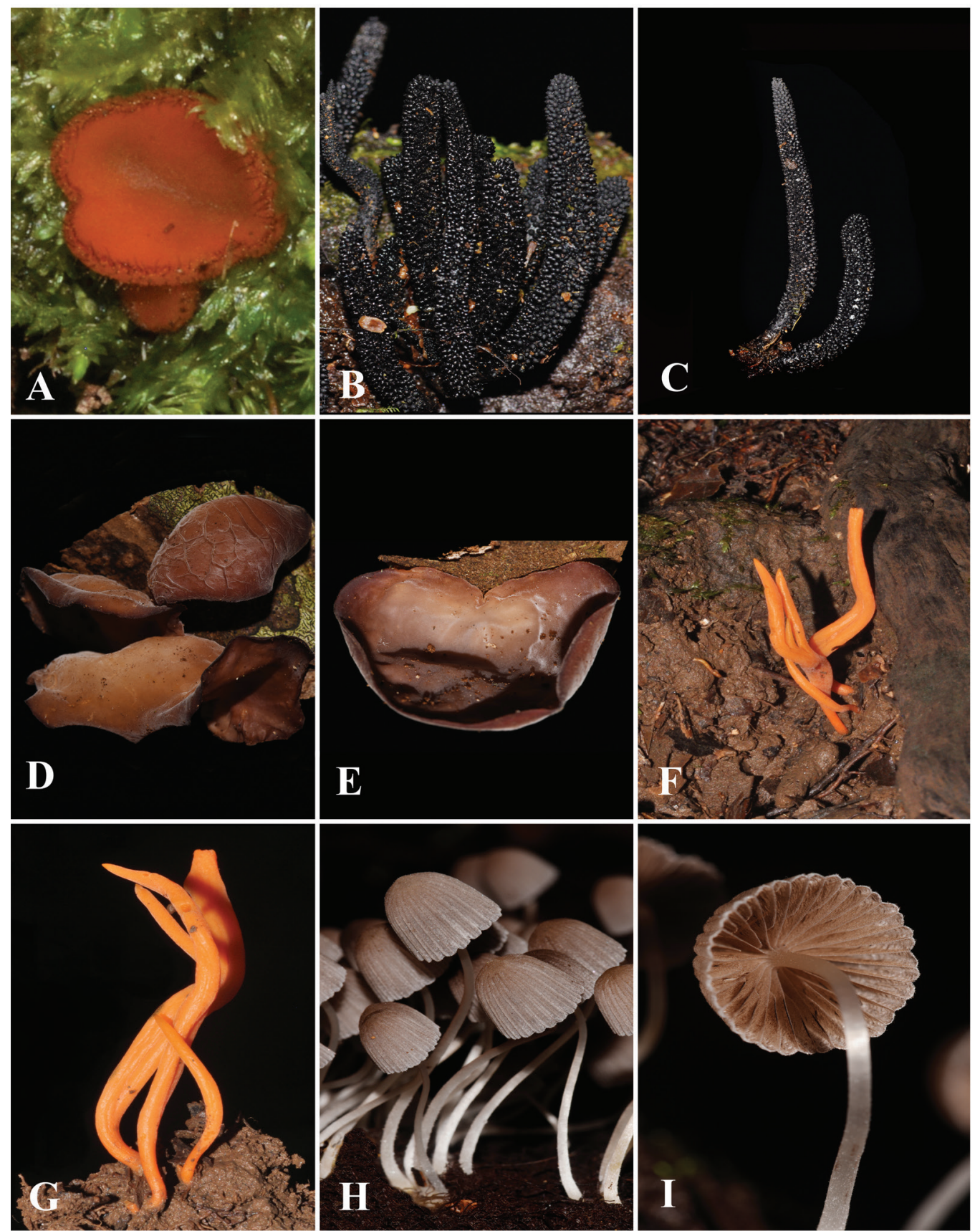

Fig. 2. Ascomycota. (A) Scutellinia sp. (A. Rodríguez 14758, CR); (B-C) Xylobotryum sp. (A. Rodríguez 14785, CR). Basidiomycota. (D-E) Auricularia auricula-judae (A. Rodríguez 14776, CR); (F-G) Clavulinopsis fusiformis (A. Rodríguez 14808, CR); (H-I) Coprinellus disseminatus (s.n.). Fotografías/Photographs: A. Rodríguez: (A-I). 


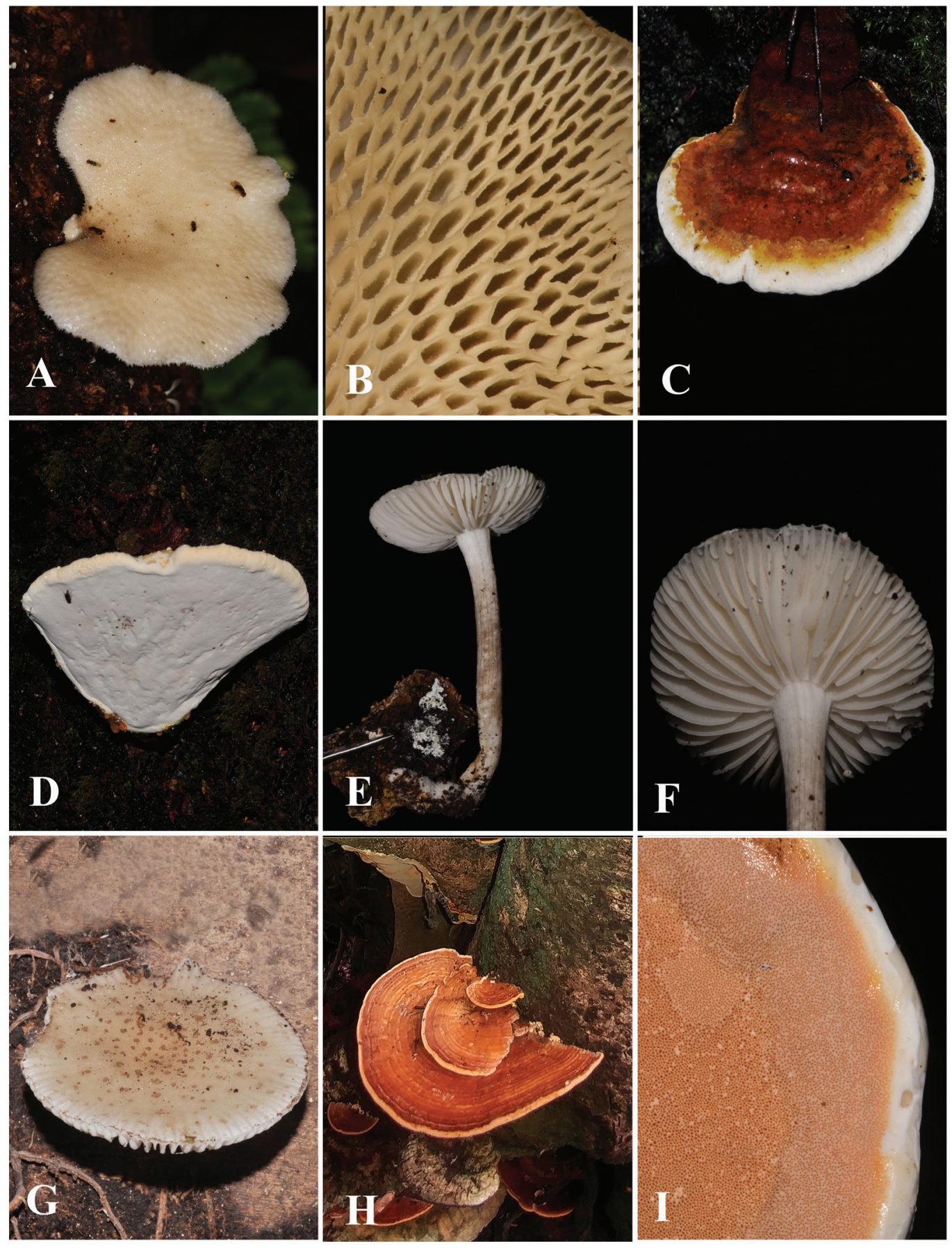

Fig. 3. Basidiomycota. (A-B) Favolus tenuiculus (A. Rodríguez 14819, CR); (C-D) Ganoderma cf. lucidum (A. Rodríguez 14783, CR); (E-G) Oudemansiella canarii (A. Rodríguez 14774, CR); (H-I) Rigidoporus microporus [(H) A. Estrada 6155, CR; (I) A. Rodríguez 14770, CR]. Fotografías/Photographs: A. Rodríguez: (A, B, C, D, E, F, G, I); A. Estrada: (H). 

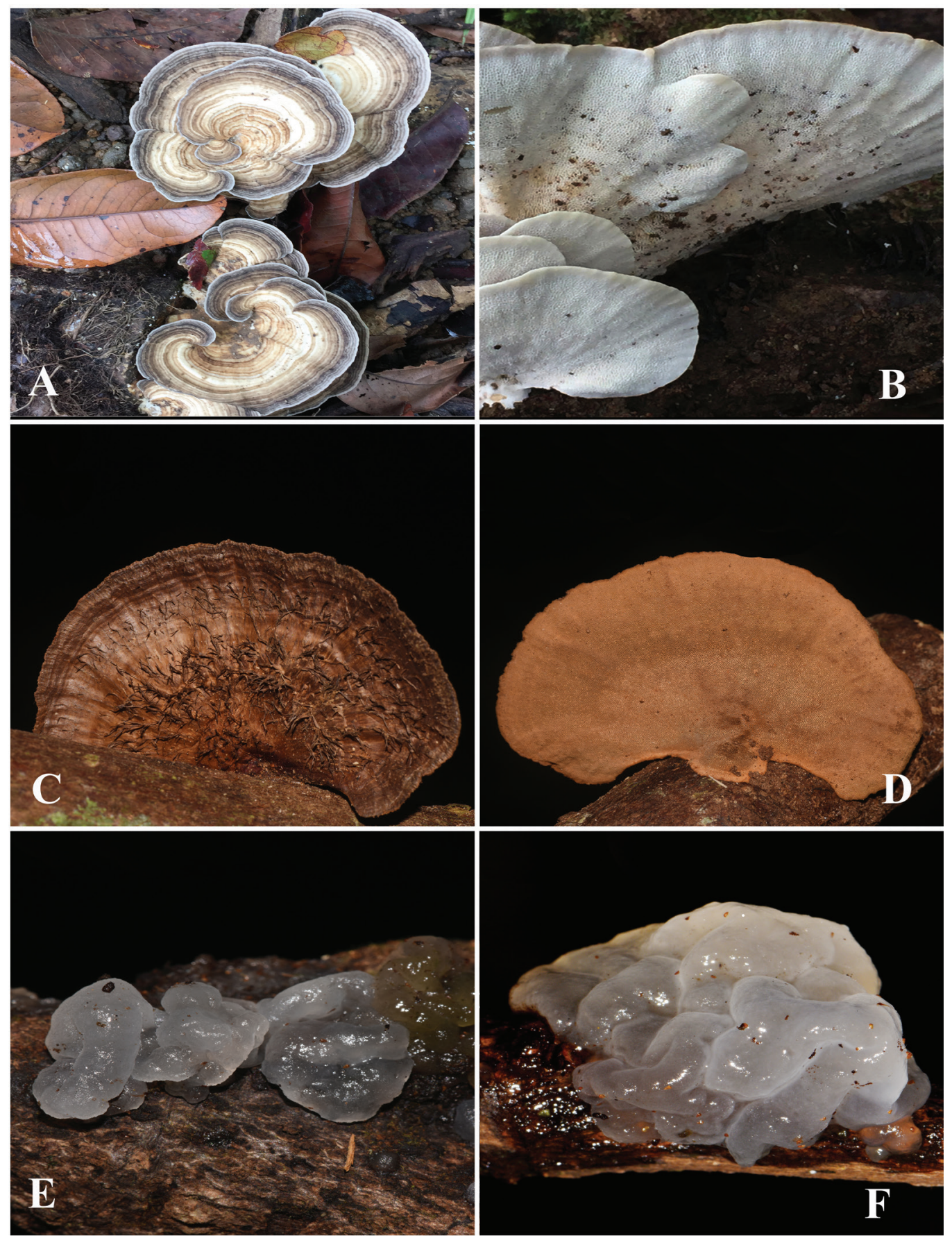

Fig. 4. Basidiomycota. (A-B) Trametes elegans (J. Sánchez 2702, CR); (C-D) Trametes hydnoides (A. Rodríguez 14821, CR); (E-F) Tremella fuciformis (A. Rodríguez 14812, CR). Fotografías/Photographs: J. Sánchez: (A-B); A. Rodríguez: (C-F). 
\& Vanegas, 2017), en la Isla del Coco se han registrado algunas especies de hongos y mixomicetos que son relevantes por sus propiedades comestibles y/o medicinales. Entre los hongos comestibles están Auricularia delicata, A. fuscosuccinea, Cookeina speciosa, Coprinellus disseminatus (Fig. 2H, Fig. 2I), Favolus tenuiculus (Fig. 3A, Fig. 3B), Oudemansiella canarii (Fig. 3E, Fig. 3F, Fig. 3G) y Tremella fuciformis (Fig. 4E, Fig. 4F); mientras que en mixomicetos solo se registra la especie Lycogala epidendrum. Asociados a la segunda condición se encuentran los hongos Trametes hydnoides (Fig. 4C, Fig. 4D), Ganoderma cf. applanatum, G. cf. lucidum (Fig. 3C, Fig. 3D), Gymnopus montagnei y Xylaria polymorpha, así como el mixomiceto Lycogala epidendrum. Esta última especie según Gamboa-Trujillo, Grefa, Uwinjin, Piyaguaje, \& Cavalcanti (2011), citado por Rodríguez-Palma et al. (2017), además de ser comestible también ha sido usada como medicinal en algunas regiones de Ecuador. Sin embargo, de acuerdo con C. Rojas (com. pers., 2018) esta información es relativamente nueva y por ahora no está bien revisada. En el caso del hongo Trametes hydnoides (Polyporaceae, Basidiomycota), Guzmán (1997) menciona que se ha empleado en México para aliviar erupciones, verrugas o infecciones en la piel. Por otra parte, algunas especies del género Ganoderma (Ganodermataceae) han sido tradicionalmente utilizadas en la medicina oriental, tal es el caso de G. lucidum, debido a su efecto vigorizante, a que incrementa la longevidad, la potencia sexual, la resistencia, la recuperación de enfermedades y a que también se ha empleado en el tratamiento del cáncer (Franco-Molano et al., 2005). No obstante, es importante advertir que en la naturaleza no todos los hongos y mixomicetos son comestibles o medicinales, por lo que se recomienda no ingerirlos, si no se conoce con exactitud su identidad.

En síntesis, a pesar de las diversas expediciones efectuadas a la Isla del Coco por varios investigadores y a la información que se ha recopilado para este estudio, todavía permanecen localidades no exploradas o poco recolectadas para hongos y mixomicetos (e.g., los cerros
Pelón, Iglesias, Las Madres y Jesús Jiménez) (Fig. 1), que son zonas de difícil acceso que dificultan la recolección, preservación y mantenimiento de los ejemplares. Por lo anterior, se concluye que el listado proporcionado en este trabajo es todavía preliminar para los grupos antes referidos. Sin embargo, la lista preparada podría ser de gran utilidad para futuros inventarios o investigaciones taxonómicas en la isla. Permitirá identificar rápidamente taxones que no han sido registrados, los cuales podrán incrementar el número de especies inventariadas o generar nuevos descubrimientos. Asimismo, se podrá detectar grupos taxonómicos poco registrados (e.g., los microhongos) o no registrados (e.g., las royas y carbones), que sin lugar a dudas deben estar presentes en la isla, que justifiquen nuevos esfuerzos de recolección enfocados mayormente al estudio de estos y otros grupos específicos.

Declaración de ética: los autores declaran que todos están de acuerdo con esta publicación y que han hecho aportes que justifican su autoría; que no hay conflicto de interés de ningún tipo; y que han cumplido con todos los requisitos y procedimientos éticos y legales pertinentes. Todas las fuentes de financiamiento se detallan plena y claramente en la sección de agradecimientos. El respectivo documento legal firmado se encuentra en los archivos de la revista.

\section{AGRADECIMIENTOS}

Al Área de Conservación Marina Cocos (ACMC) del Sistema Nacional de Áreas de Conservación (SINAC), del Ministerio de Ambiente y Energía (MINAE) por las facilidades brindadas y por permitir realizar esta investigación en el Parque Nacional Isla del Coco. A los funcionarios del parque por su colaboración durante los recorridos en el área, en especial a Guillermo Blanco Segura. Al encargado de Investigación, Esteban Herrera Herrera, por su importante ayuda. A Julieta Carranza Velázquez y Melissa Mardones Hidalgo de la Universidad de Costa Rica, por sus valiosas sugerencias y 
comentarios. A Frederick Ball por la revisión del inglés. Finalmente, al Museo Nacional de Costa Rica por proporcionar el financiamiento y apoyo logístico. Este proyecto fue llevado a cabo mediante las resoluciones del SINAC: 2015-I-ACMIC-017， 2016-I-ACMIC-016, 18-2017-I-ACMC-018.

\section{RESUMEN}

Introducción: El inventario de hongos y mixomicetos en la Isla del Coco es un proceso relativamente reciente. El primer registro de hongos fue publicado por Cooke y Bonar (1961), seguido por los listados de Fosberg y Klawe (1966), Gómez (1983) y las contribuciones de Mata (2003) y Mata, Halling, y Mueller (2003). Posteriormente a estos estudios sobre hongos, Rojas y Stevenson (2008) llevaron a cabo una investigación sobre diversidad, ecología y distribución de los mixomicetos. Objetivo: Presentar una recopilación y actualización taxonómica del inventario de hongos y mixomicetos que fueron recolectados en la Isla del Coco y facilitar el conocimiento sobre su diversidad en la isla. Métodos: La lista aquí proporcionada está basada en la revisión de la literatura disponible hasta la fecha, la consulta de bases de datos de herbarios y en la identificación de los especímenes recolectados recientemente por varios investigadores del Departamento de Historia Natural del Museo Nacional de Costa Rica. Se realizaron tres giras de campo a la isla para recolectar y fotografiar especímenes. Estas giras se llevaron a cabo entre los años 2016 y 2017, en los meses de junio, noviembre y diciembre. Los nombres de las especies incluidas se ajustaron de acuerdo con la base de datos en línea Index Fungorum. Resultados: Un total de 648 registros distribuidos en 401 hongos (principalmente macrohongos) y 247 mixomicetos fueron analizados para la Isla del Coco. Este número de registros representa 185 especies, de las cuales 139 corresponden a hongos y 46 a mixomicetos. Los hongos registrados se distribuyeron en 85 géneros, 46 familias (cuatro de ellas bajo el término "Incertae sedis"), 21 órdenes (dos de ellos como "Incertae sedis") y dos divisiones (Ascomycota y Basidiomycota); mientras tanto, los mixomicetos se clasificaron en 21 géneros, 10 familias, 5 órdenes y una división (Myxomycota). La familia mejor representada en Ascomycota fue Xylariaceae (8 spp.), seguida por Pyronemataceae (3 spp.) y Sarcoscyphaceae (2 spp.). En Basidiomycota, las familias más diversas fueron Omphalotaceae y Polyporaceae (ambas tienen $11 \mathrm{spp}$.), seguidas por Marasmiaceae (10 spp.), Agaricaceae (8 spp.), Hymenochaetaceae (8 spp.), Mycenaceae (8 spp.), Ganodermataceae (6 spp.) y Meripilaceae (6 spp.). Las familias con mayor número de especies registradas en Myxomycota fueron Stemonitidaceae (14 spp.), Physaraceae (8 spp.), Trichiaceae (6 spp.), Didymiaceae (5 spp.) y Arcyriaceae (4 spp.). Treinta y cuatro especies se registran por primera vez para la Isla del Coco, la mayor parte de ellas pertenecen a hongos (Ascomycota, 8 spp. y Basidiomycota, 21 spp.) y las restantes especies (5) a mixomicetos. En la isla algunas especies registradas son relevantes por sus propiedades comestibles o medicinales. Se incluyen fotografías de algunos de los hongos que fueron recolectados en la isla. Conclusiones: La lista generada en este estudio es todavía preliminar para los hongos y mixomicetos que se han registrado en la Isla del Coco, debido a que ciertos grupos como los microhongos han sido poco recolectados.

Palabras clave: Ascomycota; Basidiomycota; Myxomycota; neotrópico; Isla del Coco; inventario.

\section{REFERENCIAS}

Ávalos, G. (2019). Still searching the rich coast: Biodiversity of Costa Rica, numbers, processes, patterns, and challenges. En T. Pullaiah (Ed.). Global Biodiversity Volume 4: Selected countries in the Americas and Australia (pp. 101-138). Florida, USA: Apple Academic Press, CRC.

Carranza, J., \& Ruiz-Boyer, A. (2005). Checklist of polypores of Costa Rica. Revista Mexicana de Micología, 20, 45-52.

Carranza-Velázquez, J., Marín-Méndez, W., Ruiz-Boyer, A., \& Di Stéfano-Gandolfi, J. F. (2014). Riqueza de macrohongos en la Estación La Leona, Parque Nacional Corcovado, Puntarenas, Costa Rica. Brenesia, $81-82,37-51$.

Carranza-Velázquez, J., Marín-Méndez, W., Ruiz-Boyer, A., \& Di Stéfano-Gandolfi, J. F. (2017). Guía de los macrohongos más comunes del Parque Nacional Corcovado, Estación La Leona. San José, Costa Rica: UCR.

Chaverri, P., Huhndorf, S., Rogers, J., \& Samuels, G. (2010). Microhongos comunes de Costa Rica y otras regiones tropicales (Ascomycota, Pezizomycotina, Sordariomycetes). Common microfungi of Costa Rica and other tropical regions (Ascomycota, Pezizomycotina, Sordariomycetes). Santo Domingo, Heredia, Costa Rica: INBio.

Cooke, W. B., \& Bonar, L. (1961). Additional fungi from the Galapagos and other pacific coastal islands collected during the Templeton Crocker expedition, 1932. Occasional Papers of the California Academy of Sciences, 29, 1-5.

Decock, C., \& Ryvarden, L. (2013). Neotropical Perenniporia: a new species, Perenniporia subovoidea, from Costa Rica, new records of little known species, and a key to the species with a resupinate basidiome. Plant Ecology and Evolution, 146 (2), 234-239. 
Estrada, A., Sánchez, J., \& Rodríguez, A. (En prep.). Catálogo actualizado de las plantas vasculares de la Isla del Coco. Revista de Biología Tropical, este volumen.

Fosberg, F. R., \& Klawe, W. L. (1966). Preliminary list of plants from Cocos Island. In R. S. Bowman (Ed.) The Galápagos International Scientific (pp. 187189). Berkeley, USA: University of California Press.

Franco-Molano, A. E., Vasco-Palacios, A. M., López-Quintero, A. A., \& Boekhout, T. (2005). Macrohongos de las Región del medio Caquetá-Colombia. Guía de Campo: Grupo Taxonomía y Ecología de Hongos. Medellín, Colombia: Multimpresos Ltda.

Gamboa-Trujillo, P., Grefa, G., Uwinjin, P., Piyaguaje, D., \& Cavalcanti, L. H. (2011). Myxomycetes of Ecuador: Ethnomycological notes. Abstracts of the VII International Congress on Systematic and Ecology of Myxomycetes, Recife, Brasil.

Gómez, L. D. (1983). The fungi of Cocos Island, Costa Rica. Brenesia, 21, 355-364.

Guzmán, G. (1997). Los nombres de los hongos y lo relacionado con ellos en América Latina: Introducción a la Etnomicobiota y Micología aplicada de la región. Sinonimia vulgar y cientifica. Xalapa, México: Instituto de Ecología, A.C.

Guzmán, G., \& Piepenbring, M. (2011). Los hongos de Panamá: Introducción a la identificación de los macroscópicos. Xalapa, México: Instituto de Ecología, A.C.

Hammel, B. E., Grayum, M. H., Herrera, C., \& Zamora, N. (Eds.). (2004). Manual de Plantas de Costa Rica. vol. I. Introducción/Introduction. Monographs in Systematic Botany from the Missouri Botanical Garden, 97, 1-299.

Herrera, W. (1985). Clima de Costa Rica. Vol. 2. En L. D. Gómez P. (Ed.), Vegetación y clima de Costa Rica. San José, Costa Rica: EUNED.

Holmgren, P. K., Holmgren, N. H., \& Barnett, L. C. (1990). Index Herbariorum. Part I. The Herbaria of the World. 8th. Ed. Regnum vegetabile 120: 1-693.

Index Fungorum. (2018). An international project to index all formal names in the Fungi kingdom. Recuperado de http://www.indexfungorum.org/Names/ Names.asp.

Mata, M. (2003). Macrohongos de Costa Rica. 2 ed. Santo Domingo, Heredia, Costa Rica: INBio.

Mata, M., Halling, R., \& Mueller, G. (2003). Macrohongos de Costa Rica. Santo Domingo, Heredia, Costa Rica: INBio.
Mata, M., Ruiz-Boyer, A., Carranza, J., \& Ryvarden, L. (2007). Nuevos registros de hongos poliporoides (Basidiomycetes) para Costa Rica. Boletín de la Sociedad Micológica de Madrid, 31, 123-129.

Montoya, M. (1990). Plan de manejo Parque Nacional Isla del Coco. San José, Costa Rica: Sistema de Parques y Reservas Marinas (SIPAREMA), Servicio de Parques Nacionales (SPN), Ministerio de Recursos Naturales, Energía y Minas (MIRENEM). 104 pp.

Montoya, M. (2005). Salamanquesa de la Isla del Coco. El Financiero 508 (4-10 abril 2005): 30. Pausa ecológica. Organización para Estudios Tropicales.

Montoya, M. (2007). Conozca la Isla del Coco. Una guía para su visitación. Organización para Estudios Tropicales, San José, Costa Rica.

Piepenbring, M., \& Ruiz-Boyer, A. (2008). Diversity and ecology of fungi in the Golfo Dulce Region. En A. Weissenhofer, W. Huber, V. Mayer, S. Pamperl, A. Weber, y G. Aubrecht (Eds.), Natural and Cultural History of the Golfo Dulce Region, Costa Rica (pp. 179-192). Stapfia, 88. Linz, Austria: Biologiezentrum.

Rodríguez-Palma, M. M., Montoya, A., Kong, A., \& Vanegas, R. (2017). The edibility of Reticularia lycoperdon (Myxomycetes) in Central Mexico. Journal of Food Science and Nutrition, 3, 25.

Rojas, C., \& Stephenson, S. L. (2008). Myxomycete ecology along an elevation gradient on Cocos Island, Costa Rica. Fungal Diversity, 29, 117-127.

Rojas, C., Rojas, P. A., \& Lado, C. (2018). Myxomycete diversity in Costa Rica. Mycosphere, 9(2), 227-255.

Rose, J. N. (1892). List of plants from Cocos Island. In Reports on collections, and miscellaneous papers, 135. Contributions from the United States National Herbarium. Washington, DC: Government Printing Office.

Stephenson, S. L., \& Stempen, H. (1994). Myxomycetes: a handbook of slime molds. Portland, Oregon, U.S.A: Timber Press.

Stephenson, S. L., Schnittler, M., \& Novozhilov, Y. K. (2008). Myxomycete diversity and distribution from the fossil record to the present. Biodiversity and Conservation, 17, 285-301.

Tosi, J. A., Jr. (1969). Mapa ecológico: República de Costa Rica según la clasificación de zonas de vida del mundo de L. R. Holdridge. San José, Costa Rica: Centro Científico Tropical. 


\section{APÉNDICE}

\section{Lista preliminar de hongos (Ascomycota y Basidiomycota) y mixomicetos (Myxomycota) de la Isla del Coco, Puntarenas, Costa Rica}

Armando Ruiz-Boyer ${ }^{*}$ \& Alexánder Rodríguez-González ${ }^{1}$

1. Departamento de Historia Natural, Museo Nacional de Costa Rica, San José, Costa Rica; aruiz@museocostarica.go.cr, arodriguez@museocostarica.go.cr

* Correspondence

\section{Abreviaciones, códigos empleados y referencias}

Las especies que se listan a continuación se presentan en orden alfabético, según división, orden, familia y género de acuerdo con lo propuesto en la base de datos en línea "Index Fungorum" (2018). Sinonimia: (Sin.), cuando se presenta se indica entre paréntesis cuadrados posterior al nombre actual de la especie. Testigos: (CR), Herbario Nacional de Costa Rica; (F), Museo Field de Historia Natural, Chicago, Estados Unidos; (CAS), Herbario de la Academia de Ciencias de California, Estados Unidos. Referencias: las especies comunicadas por Cooke y Bonar (1961), Fosberg y Klawe (1966), Gómez (1983), Mata (2003), Mata et al. (2003) y Rojas y Stephenson (2008) se identifican con los números en superíndice 1, 2, 3, 4, 5 y 6, respectivamente. Los taxones no señalados con dichos números e identificados a nivel de especie corresponden a nuevos registros para la isla.

\section{CHAETOSPHAERIALES}

\section{ASCOMYCOTA}

\section{Chaetosphaeriaceae}

Chaetosphaeria raciborskii (Penz. \& Sacc.) F. A. Fernández \& Huhndorf / E. Fletes 7765 (CR) / 2 ejemplares.

\section{INCERTAE SEDIS}

Incertae sedis

Rimaconus jamaicensis (Seaver) Huhndorf, F. A. Fernández, Joanne E. Taylor \& K.D. Hyde / E. Fletes 7618 (CR) / 1 ejemplar.

\section{PEZIZALES}

Pezizaceae

Iodophanus

carneus (Pers.) Korf [Sin. Ascophanus carneus (Pers.) Boud.] / L. Gómez s.n. / 1 ejemplar. ${ }^{3}$

\section{Pyronemataceae}

Acervus

flavidus (Berk. \& M. A. Curtis) Pfister [Sin. Phaedropezia flavida (Berk. \& M. A. Curtis) Le Gal] / L. Gómez s.n. / 1 ejemplar. ${ }^{3}$

\section{Scutellinia}

cubensis (Berk. \& M. A. Curtis) Gamundí / L. Gómez s.n. / 1 ejemplar. ${ }^{3}$ sp. / A. Rodríguez 14758 (CR) / 1 ejemplar.

\section{Sarcoscyphaceae}

\section{Cookeina}

colensoi (Berk.) Seaver / L. Gómez s.n. / 1 ejemplar. ${ }^{3}$

speciosa (Fr.) Dennis / L. Gómez s.n. / 1 ejemplar. ${ }^{3}$ 


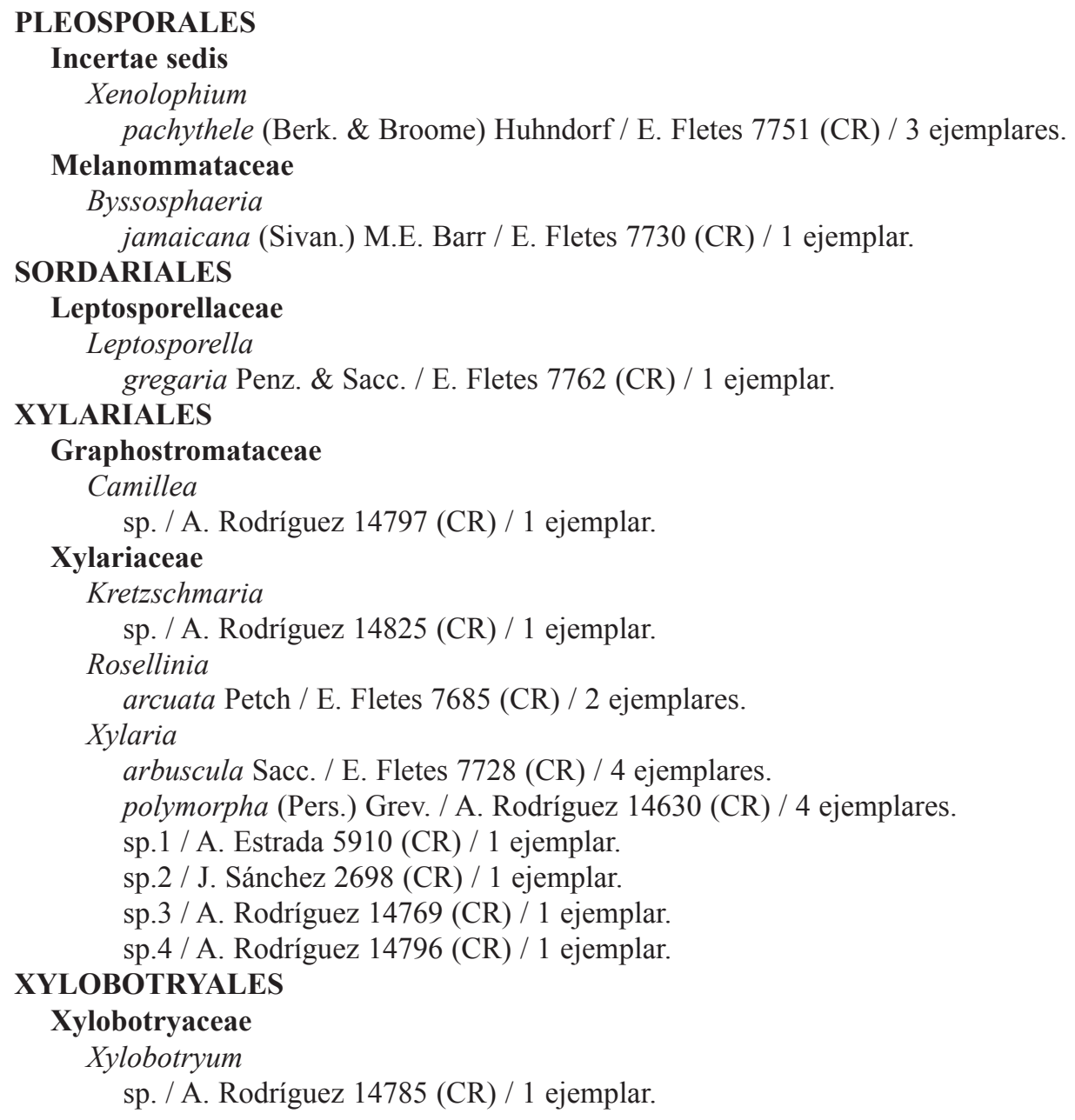

\section{BASIDIOMYCOTA}

\section{AGARICALES}

Agaricaceae

Cyathus

montagnei Tul. \& C. Tul. / L. Gómez s.n. / 1 ejemplar. ${ }^{3}$

stercoreus (Schwein.) De Toni / L. Gómez s.n. / 1 ejemplar. ${ }^{3}$

Lepiota

gracilis Peck / L. Gómez s.n. / 1 ejemplar. ${ }^{3}$

sp. 1 / L. Gómez s.n. / 1 ejemplar. ${ }^{3}$

sp. 2 / L. Gómez s.n. / 1 ejemplar. ${ }^{3}$

Lycogalopsis

solmsii E. Fisch. / E. Fletes 7624 (CR) / 3 ejemplares.

Lycoperdon

fuligineum Berk. \& M. A. Curtis / E. Fletes 7642 (CR) / 1 ejemplar.

velutinum Berk. \& M. A. Curtis [Sin. Morganella velutina (Berk. \& M. A. Curtis) Kreisel \& Dring] / E. Fletes 7717 (CR) / 2 ejemplares. ${ }^{3}$ 


\section{Amanitaceae}

Amanita

aff. antillana Dennis / L. Gómez S.n. / 1 ejemplar. ${ }^{3}$

\section{Clavariaceae}

Clavulinopsis

fusiformis (Sowerby) Corner / A. Rodríguez 14808 (CR) / 1 ejemplar.

laeticolor (Berk. \& M. A. Curtis) R. H. Petersen / L. Gómez 18032 (F) / 1 ejemplar.

\section{Hygrophoraceae}

Hygrocybe hondurensis Murrill / L. Gómez s.n. / 1 ejemplar. ${ }^{3}$

\section{Incertae sedis}

Camarophyllopsis dennisiana (Singer) Arnolds [Sin. Hygrotrama dennisianum Singer] / L. Gómez s.n. / 1 ejemplar. ${ }^{3}$

\section{Panaeolus}

aff. antillarum (Fr.) Dennis / L. Gómez s.n. / 1 ejemplar. ${ }^{3}$

semiovatus (Sowerby) S. Lundell \& Nannf. [Sin. Anellaria semiovata (Sowerby) A. Pearson \& Dennis] / L. Gómez s.n. / 1 ejemplar. ${ }^{3,4}$

\section{Inocybaceae}

Pleuroflammula

squarrulosa Singer / L. Gómez 17998 (F) / 2 ejemplares.

\section{Lycoperdaceae}

Apioperdon

pyriforme (Schaeff.) Vizzini [Sin. Lycoperdon pyriforme Schaeff.] / L. Gómez s.n. / 1 ejemplar. ${ }^{3,4}$

\section{Marasmiaceae}

Gerronema

sp.1 / L. Gómez s.n. / 1 ejemplar. ${ }^{3}$

sp. 2 / L. Gómez s.n. / 1 ejemplar. ${ }^{3}$

Hydropus

sp. / L. Gómez s.n. / 1 ejemplar. ${ }^{3}$

Lactocollybia

sp. / L. Gómez s.n. / 1 ejemplar. ${ }^{3}$

Marasmius

crinis-equi F. Muell. ex Kalchbr. [Sin. Marasmius equicrinis F. Muell. ex Berk.] / L. Gómez s.n. / 1 ejemplar. ${ }^{3}$

guyanensis Mont. / L. Gómez s.n. / 1 ejemplar. ${ }^{3}$

haematocephalus (Mont.) Fr. / L. Gómez s.n. / 1 ejemplar. ${ }^{3}$

rotuloides Dennis / L. Gómez s.n. / 1 ejemplar. ${ }^{3}$

sp. / A. Rodríguez 14780 (CR) / 1 ejemplar.

Tetrapyrgos

nigripes (Fr.) E. Horak [Sin. Marasmiellus nigripes (Fr.) Singer] / E. Fletes 7708 (CR) / 2 ejemplares. 3,4

\section{Mycenaceae}

Favolaschia sprucei (Berk.) Singer / L. Gómez s.n. / 1 ejemplar. ${ }^{3}$

Filoboletus

gracilis (Klotzsch ex Berk.) Singer / L. Gómez s.n. / 1 ejemplar. ${ }^{3}$ 


\section{Mycena}

alphitophora (Berk.) Sacc. [Sin. Mycena osmundicola J.E. Lange] / L. Gómez s.n. / 1 ejemplar. ${ }^{3}$

citricolor (Berk. \& M. A. Curtis) Sacc. / L. Gómez s.n. / 1 ejemplar. ${ }^{3}$

holoporphyra (Berk. \& M. A. Curtis) Singer / L. Gómez s.n. / 1 ejemplar. ${ }^{3}$

aff. parabolica (Fr.) Quél. / L. Gómez s.n. / 1 ejemplar. ${ }^{3}$

plectophylla (Mont.) Dennis / L. Gómez s.n. / 1 ejemplar. ${ }^{3}$

Panellus

sp. / [= Dictyopanus aff. pusillus (Pers. ex Lév.) Singer sensu Gómez (1983)] / 1 ejemplar. ${ }^{3}$

\section{Omphalotaceae}

Gymnopus

coracicolor (Berk. \& M. A. Curtis) J. L. Mata [Sin. Collybia coracicolor (Berk. \& M. A. Curtis) Dennis] / L. Gómez s.n. / 1 ejemplar. ${ }^{3}$

montagnei (Berk.) Redhead [Sin. Caripia montagnei (Berk.) Kuntze] / L. Gómez s.n. / 1 ejemplar. ${ }^{3}$

sp. / A. Rodríguez 14764 (CR) / 1 ejemplar.

Marasmiellus

cocosensis Singer / L. Gómez 17974 (F) / 2 ejemplares.

cubensis (Berk. \& M. A. Curtis) Singer / L. Gómez s.n. / 1 ejemplar. ${ }^{3}$

distantifolius (Murrill) Singer / L. Gómez s.n. / 1 ejemplar. ${ }^{3}$

echinocephalus Singer / L. Gómez s.n. / 1 ejemplar. ${ }^{3}$

pernambucensis Singer / L. Gómez 17969 (F) / 1 ejemplar.

sp.1 / L. Gómez s.n. / 1 ejemplar. ${ }^{3}$

sp. 2 / L. Gómez s.n. $/ 1$ ejemplar. ${ }^{3}$

sp. 3 / L. Gómez s.n. 11 ejemplar. ${ }^{3}$

Physalacriaceae

Flammulina

sp. / A. Rodríguez 14779 (CR) / 1 ejemplar.

Oudemansiella

canarii (Jungh.) Höhn. / A. Rodríguez 14774 (CR) / 3 ejemplares. ${ }^{3,4}$

Physalacria

clusiae Syd. / L. Gómez s.n. / 1 ejemplar. ${ }^{3}$

\section{Pleurotaceae}

Pleurotus

sp. / A. Rodríguez 14763 (CR) / 1 ejemplar.

\section{Pluteaceae}

Volvariella

pseudovolvacea (Berk. \& Broome) Singer / L. Gómez s.n. / 1 ejemplar. ${ }^{3}$

\section{Psathyrellaceae}

Coprinellus

disseminatus (Pers.) J. E. Lange [Sin. Coprinus disseminatus (Pers.) Gray] / E. Fletes 7665 (CR) / 2 ejemplares. ${ }^{3}$

\section{Tricholomataceae}

Clitocybe

microspora Peck / L. Gómez s.n. / 1 ejemplar. ${ }^{3}$

Collybia

sp. / A. Rodríguez 14627 (CR) / 1 ejemplar. 


\section{Omphalina}

smaragdina (Berk.) Malençon \& Bertault [Sin. Marasmius smaragdinus (Berk.) Singer] / L. Gómez s.n. / 1 ejemplar. ${ }^{3}$

\section{AURICULARIALES}

\section{Auriculariaceae}

Auricularia auricula-judae (Bull.) Quél. / A. Rodríguez 14776 (CR) / 4 ejemplares. delicata (Mont. ex Fr.) Henn. / L. Gómez s.n. / 1 ejemplar. ${ }^{3}$ fuscosuccinea (Mont.) Henn. / L. Gómez s.n. / 1 ejemplar. ${ }^{3}$

Exidiopsis galzinii (Bres.) Killerm. [Sin. Bourdotia galzinii (Bres.) Trotter] / L. Gómez s.n. / 1 ejemplar. $^{3}$

\section{CANTHARELLALES}

\section{Aphelariaceae}

Aphelaria

tropica (Mont.) Corner / L. Gómez s.n. / 1 ejemplar. ${ }^{3}$

\section{Clavulinaceae}

Clavulina

sp. / E. Fletes 7816 (CR) / 1 ejemplar.

Multiclavula

calocera (G.W. Martin) R. H. Petersen [Sin. Clavulinopsis calocera (G. W. Martin) Cor-

\section{Hydnaceae} ner] / L. Gómez s.n. / 1 ejemplar. ${ }^{3}$

\section{Hydnum}

sp. / E. Fletes 7815 (CR) / 1 ejemplar.

\section{Sistotrema}

diademiferum (Bourdot \& Galzin) Donk [Sin. Trechispora diademifera (Bourdot \& Galzin) D. P. Rogers] / L. Gómez s.n. / 1 ejemplar. ${ }^{3}$

\section{DACRYMYCETALES}

Dacrymycetaceae

\section{Calocera}

cornea (Batsch) Fr. / L. Gómez s.n. / 1 ejemplar. ${ }^{3}$

Dacryopinax

spathularia (Schwein.) G. W. Martin / L. Gómez s.n. / 1 ejemplar. ${ }^{3}$

\section{GEASTRALES}

\section{Geastraceae}

Geastrum

hariotii Lloyd / L. Gómez s.n. / 1 ejemplar. ${ }^{3}$

schweinitzii (Berk. \& M. A. Curtis) Zeller / E. Fletes 7651 (CR) / 2 ejemplares.

Sphaerobolus stellatus Tode / L. Gómez s.n. / 1 ejemplar. ${ }^{3}$

\section{GLOEOPHYLLALES}

Gloeophyllaceae

Boreostereum

sulphuratum (Berk. \& Ravenel) G. Y. Zheng \& Z. S. Bi [Sin. Stereum sulphuratum Berk. \& Ravenel] / L. Gómez s.n. / 1 ejemplar. ${ }^{3}$ 


\section{GOMPHALES}

Gomphaceae

Phaeoclavulina

cyanocephala (Berk. \& M. A. Curtis) Giachini [Sin. Ramaria cyanocephala (Berk. \& M. A. Curtis) Corner] / L. Gómez s.n. / 1 ejemplar. ${ }^{3}$

\section{HYMENOCHAETALES}

\section{Hymenochaetaceae}

Fulvifomes umbrinellus (Bres.) Y. C. Dai [Sin. Poria umbrinella Bres.] / A. Stewart 1425 (CAS) / 1 ejemplar. ${ }^{1}$

Hymenochaete damicornis (Link) Lév. / E. Fletes 7791 (CR) / 2 ejemplares. ${ }^{3}$ iodina (Mont.) Baltazar \& Gilbertoni / E. Fletes 7634 (CR) / 4 ejemplares.

Inonotus tabacinus (Mont.) G. Cunn. [Sin. Polyporus tabacinus Mont.; Cyclomyces tabacinus (Mont.) Pat.] / W. Klawe s.n. / 1 ejemplar. ${ }^{2,5}$

Phellinus fastuosus (Lév.) S. Ahmad [Sin. Fomes fastuosus (Lév.) Cooke] / L. Gómez s.n. / 1 ejemplar. ${ }^{3}$ gilvus (Schwein.) Pat. / E. Fletes 7804 (CR) / 1 ejemplar. sp. / F. Ball 16-2009 (CR) / 1 ejemplar.

Phylloporia pectinata (Klotzsch) Ryvarden [Sin. Fomes pectinatus (Klotzsch) Gillet] / L. Gómez s.n. / 1 ejemplar. ${ }^{3}$

\section{INCERTAE SEDIS}

\section{Incertae sedis}

Cotylidia sp. / A. Rodríguez 14778 (CR) / 1 ejemplar.

\section{POLYPORALES}

\section{Fomitopsidaceae}

Daedalea dochmia (Berk. \& Broome) T. Hatt. [Sin. Fomes dochmius (Berk. \& Broome) Cooke] / L. Gómez s.n. / 1 ejemplar. ${ }^{3}$

\section{Phaeodaedalea}

incerta (Curr.) Țura, Zmitr., Wasser \& Spirin [Sin. Phaeodaedalea sprucei (Berk.) M. Fidalgo] / L. Gómez s.n. / 1 ejemplar. ${ }^{3}$

\section{Ganodermataceae}

\section{Amauroderma} schomburgkii (Mont. \& Berk.) Torrend [Sin. Amauroderma ocellatum (Berk.) Lloyd ex Wakef.] / L. Gómez s.n. / 1 ejemplar. ${ }^{3}$

\section{Ganoderma}

cf. applanatum (Pers.) Pat. [Sin. Fomes applanatus (Pers.) Fr.] / A. Estrada 5906 (CR) / 4 ejemplares. 2,3

cf. australe (Fr.) Pat. / E. Fletes 7607 (CR) / 2 ejemplares.

cf. lucidum (Curtis) P. Karst. / A. Rodríguez 14783 (CR) / 6 ejemplares.

stipitatum (Murrill) Murrill / E. Fletes 7619 (CR) / 1 ejemplar.

sp. / F. Ball 19-2009 (CR) / 1 ejemplar. 


\section{Meripilaceae}

Rigidoporus

microporus (Sw.) Overeem / A. Estrada 6155 (CR) / 7 ejemplares.

ulmarius (Sowerby) Imazeki / E. Fletes 7879 (CR) / 1 ejemplar.

vinctus (Berk.) Ryvarden [Sin. Poria vincta Berk.) Cooke] / L. Gómez s.n. / 1 ejemplar. ${ }^{3}$ sp.1 / A. Rodríguez 14757 (CR) / 1 ejemplar.

sp.2 / F. Ball 6-2009 (CR) / 1 ejemplar.

sp.3 / A. Rodríguez 14629 (CR) / 1 ejemplar.

\section{Meruliaceae}

Aquascypha

hydrophora (Berk.) D. A. Reid / L. Gómez s.n. / 1 ejemplar. ${ }^{3}$

Flaviporus

liebmannii (Fr.) Ginns / E. Fletes 7636 (CR) / 2 ejemplares.

Mycoacia $u d a$ (Fr.) Donk [Sin. Odontia uda (Fr.) Bres.?] / W. Klawe s.n. / 1 ejemplar. ${ }^{2}$

Podoscypha

\section{Polyporaceae}

venustula (Speg.) D. A. Reid [Sin. Stereum affine Lév.] / W. Klawe s.n. / 1 ejemplar. ${ }^{2}$

\section{Earliella}

scabrosa (Pers.) Gilb. \& Ryvarden [Sin. Polyporus corrugatus Pers.; Trametes corrugata (Pers.) Bres.] / E. Fletes 7594 (CR) / 4 ejemplares. ${ }^{3}$

Favolus

tenuiculus P. Beauv. [Sin. Favolus reniformis (Murrill) Sacc. \& Trotter; Favolus brasiliensis (Fr.) Fr.; Polyporus tenuiculus (P. Beauv.) Fr.] / A. Rodríguez 14819 (CR) / 5 ejemplares. $2,3,4$

\section{Lentinus}

arcularius (Batsch) Zmitr. [Sin. Polyporus arcularius (Batsch) Fr.] / L. Gómez s.n. / 1 ejemplar. ${ }^{3}$

crinitus (L.) Fr. [citada como Lentinus “cristatus" Fr. sensu Fosberg \& Klawe (1966)] / A. Estrada 5905 (CR) / 3 ejemplares. ${ }^{2,3}$

tricholoma (Mont.) Zmitr. [Sin. Polyporus cowellii Murrill; Polyporus tricholoma Mont.] / A. Rodríguez 14777 (CR) / 4 ejemplares. ${ }^{2}$

Polyporus

dictyopus Mont. / E. Fletes 7801 (CR) / 3 ejemplares.

Tinctoporellus epimiltinus (Berk. \& Broome) Ryvarden / E. Fletes 7603 (CR) / 3 ejemplares.

\section{Trametes}

cubensis (Mont.) Sacc. [Sin. Polyporus cubensis Mont.] / L. Gómez s.n. / 1 ejemplar. ${ }^{3}$ elegans (Spreng.) Fr. [Sin. Lenzites repandus Fr.] / J. Sánchez 2702 (CR) / 6 ejemplares. ${ }^{3}$ hydnoides (Sw.) Fr. [Sin. Hexagonia hydnoides (Sw.) M. Fidalgo] / A. Rodríguez 14821 (CR) / 6 ejemplares.

Wolfiporia

cocos (Schwein.) Ryvarden \& Gilb. [Sin. Poria cocos (Schwein.) F.A. Wolf.] / L. Gómez s.n. $/ 1$ ejemplar. ${ }^{3}$ 


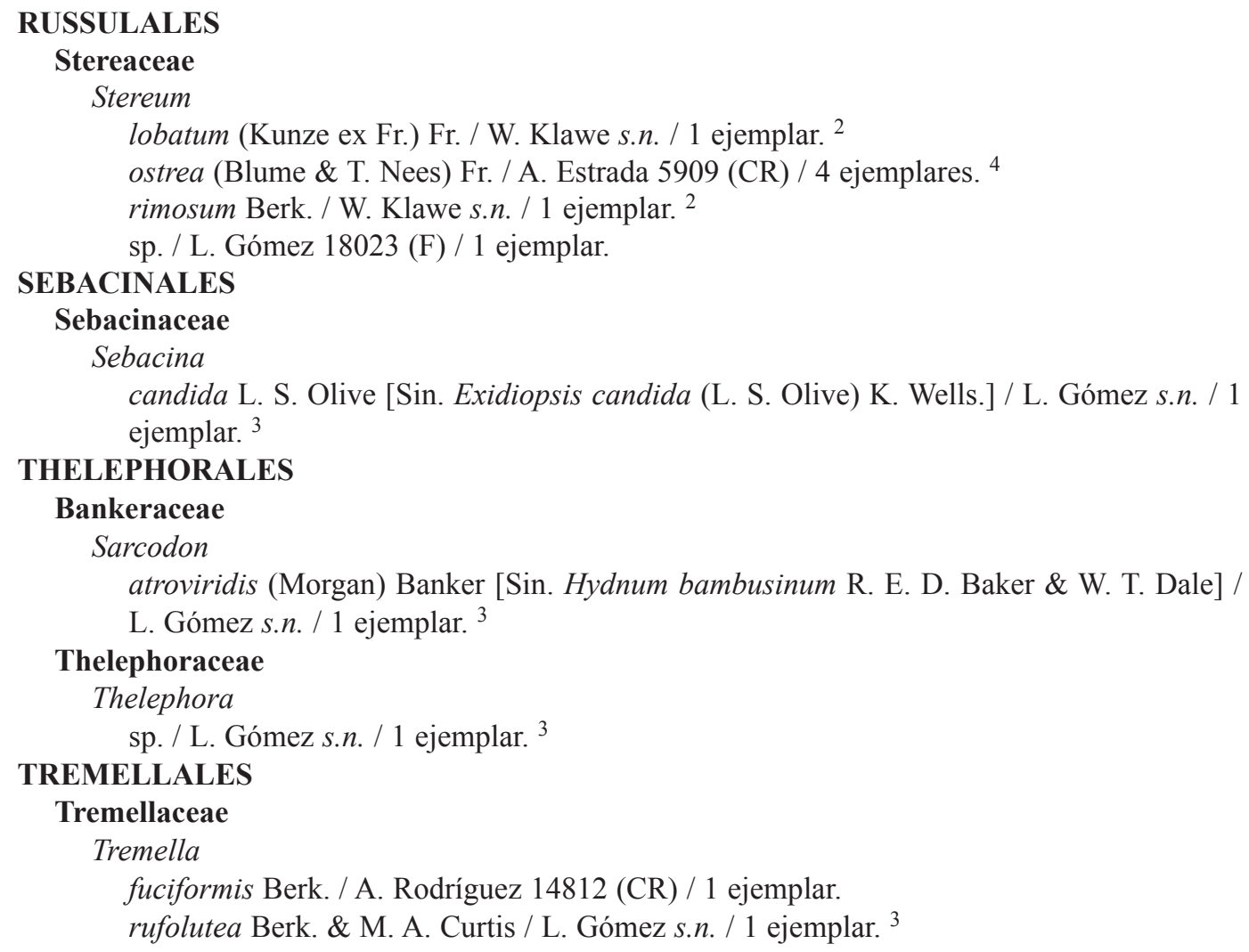

\section{THELEPHORALES}

Bankeraceae

Sarcodon

atroviridis (Morgan) Banker [Sin. Hydnum bambusinum R. E. D. Baker \& W. T. Dale] / L. Gómez s.n. $/ 1$ ejemplar. ${ }^{3}$

\section{Thelephoraceae}

Thelephora

sp. / L. Gómez s.n. / 1 ejemplar. ${ }^{3}$

\section{TREMELLALES}

Tremellaceae

Tremella

fuciformis Berk. / A. Rodríguez 14812 (CR) / 1 ejemplar.

rufolutea Berk. \& M. A. Curtis / L. Gómez s.n. / 1 ejemplar. ${ }^{3}$

\section{ECHINOSTELIALES}

\section{MYXOMYCOTA}

\section{Clastodermataceae}

Clastoderma debarynum A. Blytt / C. Rojas 942 (CR) / 5 ejemplares. ${ }^{6}$ pachypus Nann.-Bremek. / C. Rojas 885 (CR)/ 1 ejemplar. ${ }^{6}$

\section{Echinosteliaceae}

Echinostelium

minutum de Bary / C. Rojas 856 (CR) / 1 ejemplar. ${ }^{6}$

\section{LICEALES}

\section{Cribrariaceae}

Cribraria

intricata Schrad. / C. Rojas 694 (CR) / 1 ejemplar. ${ }^{6}$

microcarpa (Schrad.) Pers. / C. Rojas 959 (CR) / 17 ejemplares. ${ }^{6}$

violacea Rex / C. Rojas 838 (CR) / 8 ejemplares. ${ }^{6}$

\section{Liceaceae}

Licea

sp. / C. Rojas 879 (CR) / 1 ejemplar. ${ }^{6}$ 


\section{Tubiferaceae}

Lycogala epidendrum (J.C. Buxb. ex L.) Fr. / C. Rojas 1978 (CR) / 2 ejemplares.

Tubifera bombarda (Berk. \& Broome) G. W. Martin / C. Rojas \& S. Stephenson s.n. / 1 ejemplar. ${ }^{6}$

\section{PHYSARALES}

\section{Didymiaceae}

Diderma

effusum (Schwein.) Morgan / C. Rojas 975 (CR) / 8 ejemplares. ${ }^{6}$

hemisphaericum (Bull.) Hornem. / C. Rojas 849 (CR) / 1 ejemplar. $^{6}$

Didymium

iridis (Ditmar) Fr. / C. Rojas 787 (CR) / 1 ejemplar. ${ }^{6}$

minus (Lister) Morgan / C. Rojas 797 (CR) / 1 ejemplar. ${ }^{6}$

squamulosum (Alb. \& Schwein.) Fr. / C. Rojas 810 (CR) / 3 ejemplares. ${ }^{6}$

\section{Physaraceae}

Craterium

aureum (Schumach.) Rostaf. / C. Rojas 857 (CR) / 1 ejemplar. ${ }^{6}$

Physarella

oblonga (Berk. \& M. A. Curtis) Morgan / C. Rojas 1476 (CR) / 1 ejemplar.

Physarum

compressum Alb. \& Schwein. / C. Rojas 795 (CR) / 2 ejemplares. ${ }^{6}$

javanicum Racib. / C. Rojas 805 (CR) / 1 ejemplar. ${ }^{6}$

melleum (Berk. \& Broome) Massee / C. Rojas 686 (CR) / 4 ejemplares. ${ }^{6}$

pusillum (Berk. \& M. A. Curtis) G. Lister / C. Rojas 878 (CR) / 1 ejemplar. ${ }^{6}$

serpula Morgan / C. Rojas 831 (CR) / 2 ejemplares. ${ }^{6}$

superbum Hagelst. / C. Rojas 956 (CR) / 1 ejemplar. ${ }^{6}$

\section{STEMONITALES}

\section{Stemonitidaceae}

Collaria

arcyrionema (Rostaf.) Nann.-Bremek. ex Lado / C. Rojas 910 (CR) / 14 ejemplares. ${ }^{6}$

elegans (Racib.) Dhillon \& Nann.-Bremek ex Ing [Sin. Comatricha elegans (Racib.) G.

Lister] / C. Rojas 989 (CR) / 6 ejemplares. ${ }^{6}$

lurida (G. Lister) Nann.-Bremek / C. Rojas 809 (CR) / 7 ejemplares. ${ }^{6}$

\section{Comatricha}

laxa Rostaf. / C. Rojas 855 (CR) / 1 ejemplar. ${ }^{6}$

nigra (Pers.) J. Schröt. / C. Rojas 896 (CR) / 3 ejemplares. ${ }^{6}$

pulchella var. pulchella (C. Bab.) Rostaf. [Sin. Comatricha pulchella (C. Bab.) Rostaf.] /

C. Rojas 958 (CR) / 7 ejemplares. ${ }^{6}$

tenerrima (M. A. Curtis) G. Lister / C. Rojas 908 (CR) / 9 ejemplares. ${ }^{6}$

Diachea

leucopoda (Bull.) Rostaf. [= Diachea leucopodia (Bull.) Rostaf. sensu Rojas \& Stephenson (2008)] / C. Rojas 828 (CR) / 8 ejemplares. ${ }^{6}$

\section{Lamproderma}

arcyrioides (Sommerf.) Rostaf. / C. Rojas 982 (CR) / 5 ejemplares.

scintillans (Berk. \& Broome) Morgan / C. Rojas 952 (CR) / 5 ejemplares. ${ }^{6}$

Macbrideola

scintillans H. C. Gilbert / C. Rojas 976 (CR) / 1 ejemplar. ${ }^{6}$ 


\section{Stemonitis}

flavogenita E. Jahn / C. Rojas 841 (CR) / 1 ejemplar. ${ }^{6}$

fusca Roth / C. Rojas 929 (CR) / 6 ejemplares. ${ }^{6}$

Stemonitopsis

hyperopta (Meyl.) Nann.-Bremek. / C. Rojas 693 (CR) / 1 ejemplar. ${ }^{6}$

\section{TRICHIALES}

Arcyriaceae

Arcyria

afroalpina Rammeloo / C. Rojas 887 (CR) / 1 ejemplar. ${ }^{6}$

cinerea (Bull.) Pers. / C. Rojas 921 (CR) / 44 ejemplares. ${ }^{6}$

denudata (L.) Wettst. / S. Stephenson 18363 (CR) / 1 ejemplar.

minuta Buchet / C. Rojas 785 (CR) / 2 ejemplares. ${ }^{6}$

\section{Trichiaceae}

\section{Hemitrichia}

minor G. Lister / C. Rojas \& S. Stephenson s.n. / 1 ejemplar. ${ }^{6}$ pardina (Minakata) Ing / C. Rojas 990 (CR) / 2 ejemplares. serpula (Scop.) Rostaf. / A. Jiménez 11891 (CR) / 2 ejemplares. ${ }^{6}$

\section{Perichaena}

chrysosperma (Curr.) Lister / C. Rojas 943 (CR) / 10 ejemplares. $^{6}$

depressa Lib. / C. Rojas 808 (CR) /10 ejemplares. ${ }^{6}$

pedata (Lister \& G. Lister) G. Lister / C. Rojas 872 (CR) / 1 ejemplar. ${ }^{6}$ 